\title{
HUNTING FOR PLANETS IN THE HL TAU DISK
}

\author{
L. Testi ${ }^{1,2,3}$, A. Skemer ${ }^{4,10}$, Th. Henning ${ }^{5}$, V. Bailey ${ }^{4,11}$, D. Defrère ${ }^{4}$, Ph. Hinz ${ }^{4}$, J. Leisenring $^{4}$,

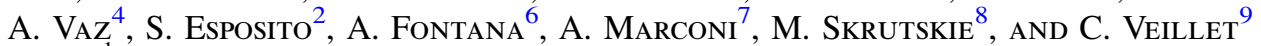 \\ ${ }^{1}$ ESO, Karl Schwarzschild str. 2, D-85748 Garching bei Muenchen, Germany; ltesti@eso.org \\ ${ }^{2}$ INAF-Osservatorio Astrofisico di Arcetri, Largo E. Fermi 5, I-50125 Firenze, Italy \\ ${ }^{3}$ Excellence Cluster "Universe", Boltzmann str. 2, D-85748 Garching bei Muenchen, Germany \\ ${ }^{4}$ Steward Observatory, University of Arizona, 933 N. Cherry Ave., Tucson, AZ 85721, USA \\ ${ }^{5}$ Max Planck Institute for Astronomie, Königstuhl 17, D-69117 Heidelberg, Germany \\ ${ }^{6}$ INAF-Osservatorio Astronomico di Roma, Monte Porzio, Italy \\ ${ }^{7}$ Universitá degli Studi di Firenze, Dipartimento di Fisica e Astronomia, Firenze, Italy \\ ${ }^{8}$ University of Virginia, 530 McCormick Road, Charlottesville, VA 22904, USA \\ ${ }^{9}$ LBT Observatory, University of Arizona, 933 N. Cherry Ave., Tucson, AZ 85721, USA \\ Received 2015 June 23; accepted 2015 September 17; published 2015 October 21
}

\begin{abstract}
Recent ALMA images of HL Tau show gaps in the dusty disk that may be caused by planetary bodies. Given the young age of this system, if confirmed, this finding would imply very short timescales for planet formation, probably in a gravitationally unstable disk. To test this scenario, we searched for young planets by means of direct imaging in the $\mathrm{L}^{\prime}$ band using the Large Binocular Telescope Interferometer mid-infrared camera. At the location of two prominent dips in the dust distribution at $\sim 70 \mathrm{AU}(\sim 0.5)$ from the central star, we reach a contrast level of $\sim 7.5 \mathrm{mag}$. We did not detect any point sources at the location of the rings. Using evolutionary models we derive upper limits of $\sim 10-15 M_{\text {Jup }}$ at $\leqslant 0.5-1$ Ma for the possible planets. With these sensitivity limits we should have been able to detect companions sufficiently massive to open full gaps in the disk. The structures detected at millimeter wavelengths could be gaps in the distributions of large grains on the disk midplane caused by planets not massive enough to fully open the gaps. Future ALMA observations of the molecular gas density profile and kinematics as well as higher contrast infrared observations may be able to provide a definitive answer.
\end{abstract}

Key words: planet-disk interactions - protoplanetary disks - stars: individual (HL Tau)

\section{INTRODUCTION}

The protoplanetary disk surrounding the HL Tau young stellar object has been extensively studied in the past and was the first to be kinematically resolved (e.g., Sargent \& Beckwith 1991). The system is known to be very young, with an envelope still accreting onto the disk (e.g., Hayashi et al. 1993; Men'shchikov et al. 1999). The age has been estimated to be $\leqslant 1 \mathrm{Ma}$, and values as low as $0.2 \mathrm{Ma}$ have been found in the literature (Guilloteau et al. 2011). With an estimated stellar mass of $\sim 0.7 M_{\odot}$ (Kenyon \& Hartmann 1995; Close et al. 1997) and a total disk mass in the range of $\sim 0.1-0.15 M_{\odot}$ (Guilloteau et al. 2011; Kwon et al. 2011), the disk may be very close to being gravitationally unstable.

The latest ALMA long baselines science verification results show a series of eccentric bright and dark rings (ALMA Partnership et al. 2015), which could be consistent with the presence of young protoplanets in the disk. The pair of dark rings named D5 and D6, with some of the lowest optical depths in the ALMA image, have radii of $\sim 65$ and $\sim 75 \mathrm{AU}(\sim 0$ !" 5$)$ from the central star. These fall in the region of the disk predicted to be gravitationally unstable by Kwon et al. (2011). If a planetary body is indeed responsible for opening the D5/ D6 gaps, given the distance from the central star, the disk properties, and the system age, the most likely formation route for such planets would be through gravitational instabilities (Helled et al. 2014). The possibility of the presence of planets

\footnotetext{
${ }^{10}$ Current address: University of California, Santa Cruz, 1156 High Street, Santa Cruz, CA 95064, USA.

${ }^{11}$ Current address: Kavli Institute of Astrophysics and Cosmology, Stanford University, Stanford, CA 94305, USA.
}

formed by gravitational instabilities can thus be tested directly with large telescopes equipped with adaptive optics (AO) and thermal infrared diffraction limited cameras.

We used the Large Binocular Telescope Interferometer midinfrared camera (LBTI/LMIRcam) to search for the presence of young giant planets within the disk of HL Tauri, specifically, but not exclusively, at the location of the D5/D6 dark rings in the ALMA image. We describe the observations in Section 2, the results are presented and compared with model predictions in Section 3, and our conclusions are summarized in Section 5.

HL Tauri is located in the Taurus molecular cloud and throughout this paper we will adopt the commonly used distance of 140 pc (Kenyon et al. 2008).

\section{OBSERVATIONS}

We observed HL Tau with the LBTI (Hinz et al. 2014) and its $1-5 \mu \mathrm{m}, \mathrm{L} / \mathrm{M}$ Infrared Camera (LMIRcam; Skrutskie et al. 2010; Leisenring et al. 2012). LBTI/LMIRcam is used with the LBT's dual deformable secondary AO systems (Esposito et al. 2011) either for interferometry or for standard AO imaging. For the observations described in this paper, we used only one side of the LBT's two $8.4 \mathrm{~m}$ diameter primary mirrors for AO imaging. We observed HL Tau on UT 2014 November 7 and UT 2014 November 17 with the $L^{\prime}(3.8 \mu \mathrm{m})$ filter and on UT 2014 November 19 with the $\mathrm{K}_{s}(2.2 \mu \mathrm{m})$ filter. Seeing during these observations was measured by an on-site

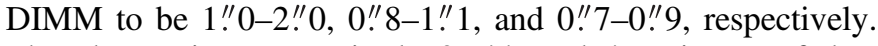
The observations comprised 70, 44, and 47 minutes of data with $59^{\circ}, 63^{\circ}$, and $59^{\circ}$ of parallactic angle rotation, respectively. 


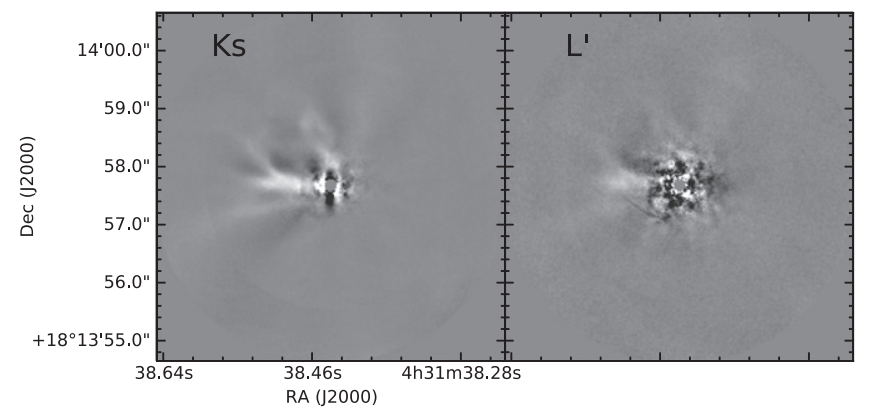

Figure 1. LBTI $\mathrm{K}_{s}$ and $\mathrm{L}^{\prime}$ images of the HL Tau system (the star is hidden by the central mask). Scattered light emission from the disk and envelope is visible. The stellar PSF has been modeled and subtracted.

HL Tau is $R \sim 14 \mathrm{mag}$, which is faint for most natural guide star AO systems, but the LBTAO system is more sensitive than other AO systems because it uses (a) a pyramid wavefront sensor and (b) on-chip detector binning to reduce the read-out noise level when operating with faint stars (Esposito \& Riccardi 2001; Esposito et al. 2011). Another option would be to guide on XZ Tau, a 0." 3 binary that is $\sim 30^{\prime \prime}$ from HL Tau. At the time of our observations, XZ Tau was $\sim 2$ mag brighter than HL Tau on the AO wavefront sensor. We used HL Tau as our guide star for the November 7 observations, and then XZ Tau for the subsequent observations. Guiding on XZ Tau produced higher Strehl ratio images of HL Tau. However, isoplanatic effects stretched the HL Tau image in the direction of the guide star.

We processed the images with the high-contrast optimization pipeline developed for the LBTI Exozodi Exoplanet Common Hunt survey (LEECH; Skemer et al. 2014; Maire et al. 2015). After basic infrared processing (nod-subtracting, registering, coadding), we model and subtract the star using angular differential imaging (Marois et al. 2006) and principle component analysis (PCA; Soummer et al. 2012; Amara \& Quanz 2012). Our final $\mathrm{K}_{s}$ and $\mathrm{L}^{\prime}$ images are shown in Figure 1. There is a clear fixed residual between the two images, which is scattered light from HL Tau's envelope, as processed by PCA, which self-subtracts (hence the negative residuals)

The structure of the scattered light envelope impacts our ability to search for point-source exoplanet companions. However, cool exoplanets are much redder than scattered starlight at these wavelengths $\left(\mathrm{K}_{s}-\mathrm{L}^{\prime} \sim 2 \mathrm{mag}\right.$ for COND/ DUSTY models at our detection threshold; Chabrier et al. 2000; Baraffe et al. 2003), so a putative exoplanet would appear as a point source in the $\mathrm{L}^{\prime}$ image, while being much fainter or undetectable in the $\mathrm{K}_{s}$ image. To search for red companions, we subtract the $\mathrm{K}_{s}$ image from the $\mathrm{L}^{\prime}$ image, ${ }^{12}$ scaled so that the scattered light envelope is suppressed as much as possible. We optimize the overall scaling and the number of principle components used to subtract the stellar point-spread function (PSF) at every radius, as described in detail in Maire et al. (2015) and briefly described in the following. Artificial point sources are inserted into the original data, one by one, at eight different azimuths per radius. For each artificial point source, we run our PCA star subtraction code, iteratively adjusting the brightness of the artificial planet until it reaches five times the standard deviation of a diffraction limit size smoothed annulus at the same radius. The number of

\footnotetext{
${ }^{12}$ This is analogous to simultaneous differential imaging (Racine et al. 1999)
}

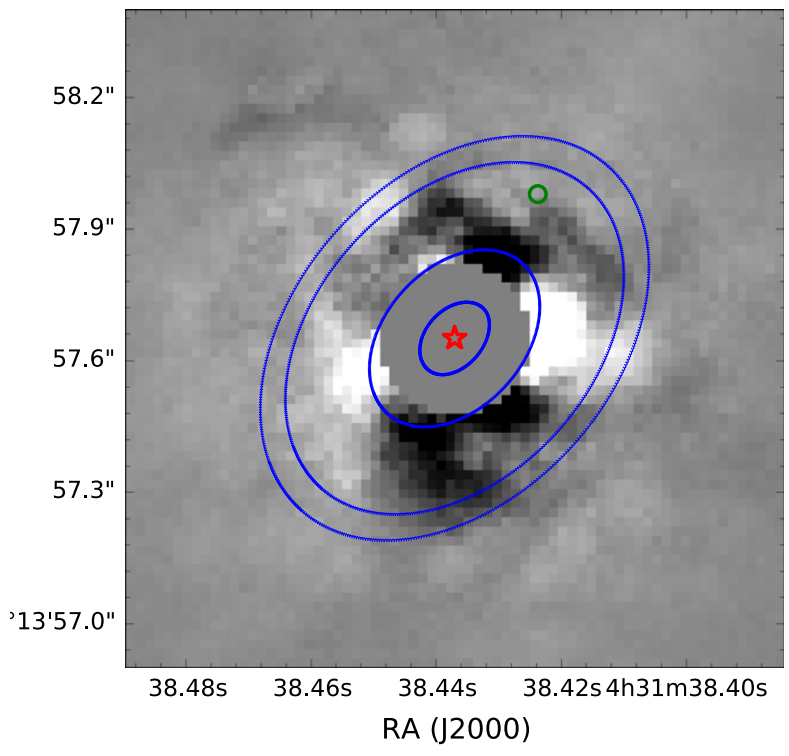

Figure 2. LBTI L' image of the HL Tau system (the star is hidden by the central mask), with scattered light emission mitigated using the $\mathrm{K}_{S}$ image as described in the text. No point source is detected in the image. The red cross marks the position of the young star. The green circle shows the position of the unconfirmed protoplanet reported by Greaves et al. (2008). The blue ellipses mark the position of the highest contrast dark rings in the ALMA image (D1, D2, D5, and D6, as defined in ALMA Partnership et al. 2015).

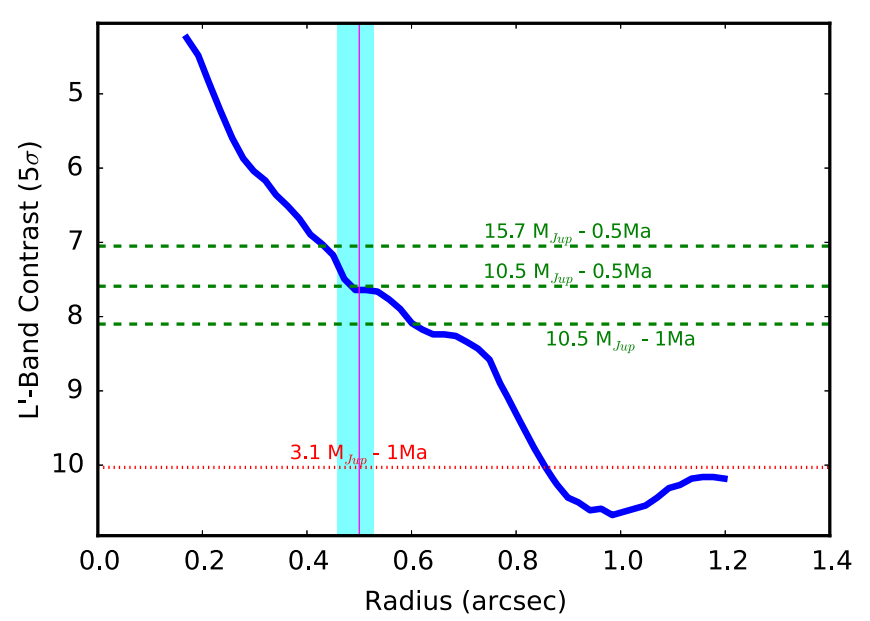

Figure 3. $\mathrm{L}^{\prime}$ contrast as a function of angular distance from HL Tau. The blue line shows the $5 \sigma$ detection limit for point sources computed from the image shown in Figure 2. The vertical cyan band marks the approximate location of the D5/D6 rings in the ALMA image. The dotted red line shows the expected emission level for a $3.1 M_{\text {Jup }}$ planet, assuming an age of $1 \mathrm{Ma}$ and the evolutionary tracks of Baraffe et al. (2008). The three green dashed lines show the expected emission for $10.5 M_{\mathrm{Jup}}$ at 0.5 and $1 \mathrm{Ma}$ and $\sim 15.7 M_{\mathrm{Jup}}$ at $0.5 \mathrm{Ma}$, as labeled, using the evolutionary tracks of Baraffe et al. (2015). See the text for details.

principle components that gives the faintest $5 \sigma$ artificial planets is used to produce the final image (Figure 2). The average magnitude of the $5 \sigma$ artificial planets at each radius is plotted as our contrast curve (Figure 3).

\section{RESULTS}

In Figure 2 we show as blue ellipses the location of the highest contrast dark rings in the ALMA images: D1, D2, D5 and D6, as defined by ALMA Partnership et al. (2015). We also 
show the location of HL Tau with a red star, and the position of the unconfirmed protoplanet reported by Greaves et al. (2008; green circle). The inner masked region has a radius of $\sim 0$ ". 18 . We do not detect any point source in the image, however this has to be interpreted with caution as the point-source sensitivity is a strong function of the distance from the central star.

In Figure 3 we show the computed $5 \sigma$ contrast limit for a point-source detection in the image of Figure 2 (see Section 2). The minimum contrasts for detection are approximately $\sim 4.5$ magnitudes at the location of D2 $(\sim 0$ !' 2$), \sim 7.5$ at the location of D5 and D6 $(\sim 0.5)$, and exceeding 10 beyond $\sim 0$ ". 85 $(\sim 120 \mathrm{AU})$. At the location of the candidate protoplanet reported by Greaves et al. (2008), the contrast limit for detection is $\sim 7$. To convert these contrast levels in an absolute magnitude limit, we need an estimate of the $\mathrm{L}^{\prime}$ magnitude of the star. Seeing-limited L-band photometry is available (e.g., Kenyon \& Hartmann 1995), but the source has a significant extended component that is well resolved in our high angular resolution images.

Close et al. (1997) estimated that the compact component of the emission in their 0 !! 2 resolution images is approximately 0.6 magnitudes fainter than the total emission at $\mathrm{K}^{\prime}$. Assuming a similar ratio for the $\mathrm{L}^{\prime}$ band and using the $(K-L)$ color from Kenyon \& Hartmann (1995), we estimate an absolute magnitude of $L \sim 0.5$ for the central point source. To obtain the intrinsic absolute magnitudes, we additionally need an estimate of the extinction. The extinction toward HL Tau has been quoted in the range 7-24 in the V band (e.g., Close et al. 1997). Using the extinction law of Rieke \& Lebofsky (1985) this corresponds to $0.4-1.3 \mathrm{mag}$ in $\mathrm{L}^{\prime}$. Here we will use a value of $\sim 1$, which needs to be subtracted from the limits derived above. Note that in this procedure we are neglecting the possible extinction provided by the disk material, which implies that the limits we are deriving strictly apply only to planets that have completely cleared a gap in the disk dust distribution.

Our final estimate of the $\mathrm{L}^{\prime}$ intrinsic absolute magnitude for the unresolved component in the HL Tau system is thus $\sim-0.5 \mathrm{mag}$. We will use this value to estimate the absolute magnitudes that correspond to our sensitivity limits by adding the value to the contrast plotted in Figure 3. The three sources of uncertainty on this estimate are the fraction of extended emission, the exact extinction value, and the possible photometric variability of the central star. To estimate the latter, we checked the WISE database and found a peak-to-peak variation of $\sim 0.4$ mag. ${ }^{13}$ Considering all these factors, we estimate an overall uncertainty of $\sim 0.5 \mathrm{mag}$.

To convert the absolute magnitudes in an estimate of the planetary companion masses, we used the evolutionary tracks of Baraffe et al. (2008, 2015). As discussed by several authors, the evolutionary tracks are very uncertain at young ages, as the initial conditions of protoplanets may depend on the formation scenario (see the discussion in Baraffe et al. 2010). Planets forming via core accretion may be expected to have cooler initial conditions, leading to smaller radii and fainter magnitudes for the same mass as compared to the canonical evolutionary tracks (Marley et al. 2007). The recent analysis by Marleau \& Cumming (2014) has shown that few directly imaged exoplanets at large radii have physical parameters

\footnotetext{
13 The WISE photometry is uncertain as HL Tau is in the saturated sources regime; the source has measured magnitudes in the range 5.0-5.4 in the WISE W1 band over a period of 200 days.
}

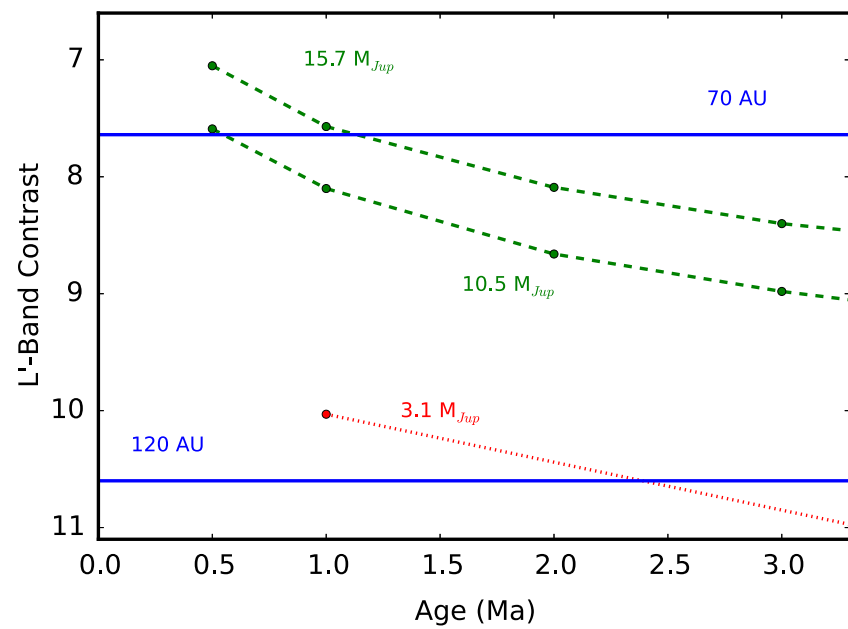

Figure 4. Contrast as a function of age for young planets with masses $3.1 M_{\mathrm{Jup}}$ from Baraffe et al. (2008; red circles and dotted lines) and 10.5 and $15.7 M_{\text {Jup }}$ from Baraffe et al. (2015; green circles and dashed lines), with the assumptions discussed in the text. Blue horizontal lines show the $5 \sigma$ detection limits for point sources at 70 and $120 \mathrm{AU}$ as derived from Figure 3.

consistent with warm initial conditions. Their results rule out cold initial conditions for the exoplanets in the HR 8799, 2MASS J12073346-3932539, and $\beta$ Pictoris systems. Warm initial conditions are thus confirmed for giant planets at large distances from the central star, and potentially also for planets formed via core accretion (as it may be the case for $\beta$ Pic b). In our case, given the young age of the system, the fact that the disk appears to be marginally unstable, and the large distances from the star we are probing with our observations, it is most likely that any large planet would have been formed via gravitational instability, rather than core accretion. We thus expect that the upper limits on the planetary masses derived from evolutionary tracks with warm initial conditions will be the most appropriate. The earliest ages for which the tracks are tabulated is $1 \mathrm{Ma}$ in Baraffe et al. (2008) and 0.5 Ma in Baraffe et al. (2015; note that these authors have $\sim 10 M_{\text {Jup }}$ as the lowest mass for their computations). As the system is believed to be younger than $0.5-1 \mathrm{Ma}$, and the possible companions even younger, it is likely that the $5 \sigma$ upper limits that we derive are conservative.

We report the results of our analysis in Figures 3 and 4. In Figure 3 we show the contrast predicted by evolutionary tracks for four different set of parameters: planets with masses $\sim 3$ and $\sim 16 M_{\text {Jup }}$ at an age of 1 Ma from Baraffe et al. (2008) and $\sim 16$ $M_{\text {Jup }}$ at $0.5 \mathrm{Ma}$ and $\sim 10.5 M_{\text {Jup }}$ at 0.5 and $1 \mathrm{Ma}$ from Baraffe et al. (2015). Given the expected age of the system and considering a $0.5 \mathrm{mag}$ uncertainty in the absolute magnitude value due to the uncertainties discussed above, planets more massive than $\sim 10-15 M_{\text {Jup }}$ should be detected if present at the location of the D5/D6 rings and at larger distances in the disks. In Figure 4 we show how planetary evolutionary tracks for 10 and $15.7 M_{\text {Jup }}$ compare with our $5 \sigma$ detection limits at 70 AU. For completeness, we also show a track for $3.1 M_{\text {Jup }}$ and the contrast limit at $120 \mathrm{AU}$, suggesting that it would be detectable in the outer disk. Nevertheless, such a planet would most likely be unable to clear a full gap in the disk and would suffer a higher extinction than our estimate (see below). 


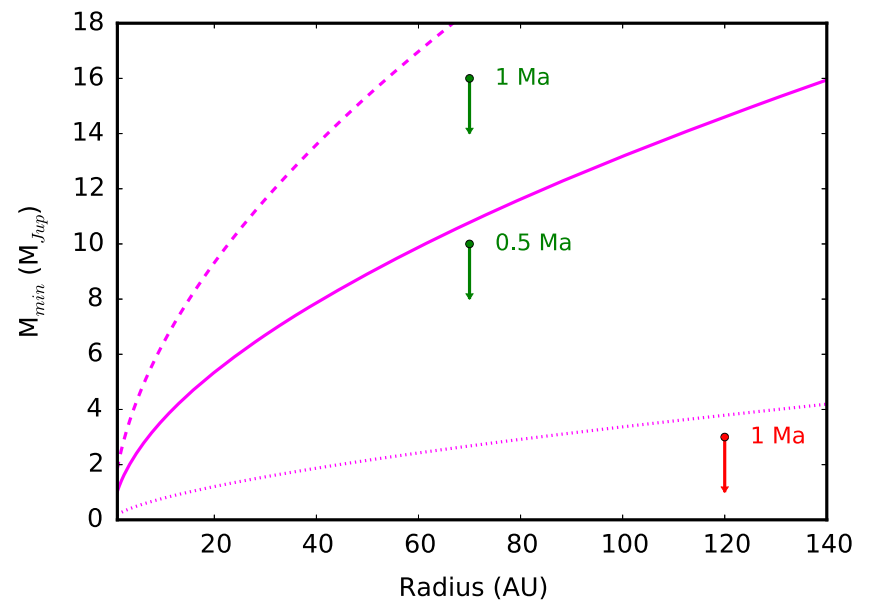

Figure 5. Gap opening criterion for the fiducial disk model discussed in the text, derived using the formula in Baruteau et al. (2014), shown as a full magenta line for the reference viscosity value $(\alpha=0.1)$. Dotted and dashed lines show the effect of reducing $(\alpha=0.001)$ or increasing $(\alpha=0.2)$ the viscosity. The $5 \sigma$ upper limits for 0.5 and $1 \mathrm{Ma}$ at $70 \mathrm{AU}$ derived from our observations are shown as green symbols, respectively. For completeness we also show our limit for $1 \mathrm{Ma}$ at $120 \mathrm{AU}$ (red symbol). See the text for details.

\section{DISCUSSION}

Using a fiducial disk model around a $\sim 1 M_{\odot}$ mass star, with a temperature profile on the midplane $T(r)=300 \mathrm{~K}$ $\times(r / 1 \mathrm{AU})^{0.5}$ and a viscosity parameter $\alpha=0.01$, we can use the formula provided in Baruteau et al. (2014) for the gap opening criterion derived by Crida et al. (2006) to estimate the minimum mass for a planet to carve a gap in the disk. The comparison of these results with our planetary mass upper limits are shown in Figure 5. At $\sim 70$ AU from the star, our upper limits are comparable to the minimum mass derived using the Crida et al. (2006) criterion, depending on the exact age of the planets. In our analysis we have assumed an age range of $0.5-1.0 \mathrm{Ma}$ for the possible planets (based on the estimated age for the star), but these would most certainly be upper limits to the age of the planets. Our estimated planetary mass upper limits are thus conservative, as younger planets would be brighter. Malik et al. (2015) recently examined the gap opening effectiveness of giant planets undergoing migration in disks and found that planets significantly above the Crida et al. (2006) criterion may still be unable to open a gap. In particular, in all their simulations a $15 M_{\text {Jup }}$ planet was unable to open a gap in the outer disk. In addition, we note that the planetary mass required to open a gap in the disk is a very strong function of the disk viscosity; for gravitationally unstable disks larger values of $\alpha$ may be appropriate.

We do not detect planets at the location of the D5/D6 gaps detected by ALMA down to the mass limits that would be expected for companions with the ability to fully clear a gap. Nevertheless, there are still possible alternatives, which involve the presence of planets, that are still plausible for the HL Tau disk. The ALMA observations reported by ALMA Partnership et al. (2015) are mostly sensitive to the distribution of the millimeter size grains. Zhu et al. (2014) have shown that even very small planetary cores are capable of creating disturbances in the gas distribution that are sufficient to efficiently trap the large grains. Dipierro et al. (2015) analyzed in more detail the case of HL Tau and showed that planets as small as $\sim 0.2-0.5$ $M_{\text {Jup }}$ may be able to explain the ALMA observations. Relatively small giant planets, smaller than our detection limit, could thus be present in the disk and produce the effects observed by ALMA if the disk viscosity is very low. For disk parameters very similar to those of HL Tau, Boss (2011) has shown that $\sim 5 M_{\text {Jup }}$ planets could form at $70 \mathrm{AU}$ through disk gravitational instabilities. Such planets, if formed very early in the disk and if they have not migrated significantly in the following 0.5-1 Ma, may have escaped our detection and, while being unable to carve proper gaps in the gaseous disk, would have sufficient mass to produce the observed confinement of the millimeter size grains.

\section{CONCLUSIONS}

We have searched for possible young giant planets in the HL Tau disk using the LBTI. We do not detect point sources within the disk in the parameter range probed by our observations. More specifically, no companions with masses above $\sim 10-15$ $M_{\text {Jup }}$ and ages $\leqslant 0.5-1 \mathrm{Ma}$ are detected at the location of the gap detected by ALMA in the dust emission at $~ 70 \mathrm{AU}$ from the star, under the assumption that such planets would have mostly cleared a gap in the vertical disk dust distribution.

Our limits imply that large planets capable of opening a full gap in the gaseous disk at $\sim 70 \mathrm{AU}$ cannot be the explanation for the distribution of dust grains as observed with ALMA. If planets are present in the HL Tau disk they are relatively small giant planets that have not opened a full gap in the disk. This conclusion is consistent with recent theoretical analysis (e.g., Dipierro et al. 2015).

There are two obvious observational avenues to further constrain the origin of the large grain confinement in HL Tau: constrain the gas distribution and properties (e.g., viscosity) and improve on our planet detection limits. The former can be attempted with ALMA, although the results of the Science Verification observations show that it will require finding tracers less affected by the envelope emission and a substantial investment of observing time. Completely excluding the presence of giant planets formed by gravitational instabilities at the location of the D5/D6 dark rings will require an improvement of $\sim 3-4$ mag in the contrast at $\sim 0$ ". 5 from the central star.

We thank the LBT Observatory director and staff for the flexibility in scheduling this project and for supporting the observations. This publication makes use of data products from the Wide-field Infrared Survey Explorer, which is a joint project of the University of California, Los Angeles, and the Jet Propulsion Laboratory/California Institute of Technology, funded by the National Aeronautics and Space Administration. The LBT is an international collaboration among institutions in the United States, Italy, and Germany. LBT Corporation partners are: The University of Arizona on behalf of the Arizona university system; Istituto Nazionale di Astrofisica, Italy; LBT Beteiligungsgesellschaft, Germany, representing the Max-Planck Society, the Astrophysical Institute Potsdam, and Heidelberg University; The Ohio State University, and The Research Corporation, on behalf of The University of Notre Dame, University of Minnesota, and University of Virginia. A.S. is supported by the National Aeronautics and Space Administration through Hubble Fellowship grant HSTHF251349 awarded by the Space Telescope Science Institute, which is operated by the Association of Universities for Research in Astronomy, Inc., for NASA, under contract NAS 5-26555. Development of the LEECH high-contrast imaging pipeline is funded by the NASA Origins of Solar Systems 
Program, grant NNX13AJ17G. The Large Binocular Telescope Interferometer is funded by the National Aeronautics and Space Administration as part of its Exoplanet Exploration program. LMIRcam is funded by the National Science Foundation through grant NSF AST-0705296. This work was partly supported by the Italian Ministero dell'Istruzione, Università e Ricerca through the grant Progetti Premiali 2012-iALMA (CUP C52I13000140001).

\section{REFERENCES}

ALMA Partnership, Brogan, C. L., Perez, L. M., et al. 2015, arXiv:1503.02649 Amara, A., \& Quanz, S. P. 2012, MNRAS, 427, 948

Baraffe, I., Chabrier, G., \& Barman, T. 2008, A\&A, 482, 315

Baraffe, I., Chabrier, G., \& Barman, T. 2010, RPPh, 73, 016901

Baraffe, I., Chabrier, G., Barman, T. S., Allard, F., \& Hauschildt, P. H. 2003, A\&A, 402, 701

Baraffe, I., Homeier, D., Allard, F., \& Chabrier, G. 2015, arXiv:1503.04107

Baruteau, C., Crida, A., Paardekooper, S.-J., et al. 2014, in Protostars and Planets VI, ed. H. Beuther et al. (Tucson, AZ: Univ. Arizona Press), 667 Boss, A. P. 2011, ApJ, 731, 74

Chabrier, G., Baraffe, I., Allard, F., \& Hauschildt, P. 2000, ApJ, 542, 464

Close, L. M., Roddier, F., Northcott, M. J., Roddier, C., \& Elon Graves, J. 1997, ApJ, 478, 766

Crida, A., Morbidelli, A., \& Masset, F. 2006, Icar, 181, 587

Dipierro, G., Price, D., Laibe, G., et al. 2015, MNRAS, 453, L73

Esposito, S., \& Riccardi, A. 2001, A\&A, 369, L9

Esposito, S., Riccardi, A., Pinna, E., et al. 2011, Proc. SPIE, 8149, 814902
Greaves, J. S., Richards, A. M. S., Rice, W. K. M., \& Muxlow, T. W. B. 2008, MNRAS, 391, L74

Guilloteau, S., Dutrey, A., Piétu, V., \& Boehler, Y. 2011, A\&A, 529, A105

Hayashi, M., Ohashi, N., \& Miyama, S. M. 1993, ApJL, 418, L71

Helled, R., Bodenheimer, P., Podolak, M., et al. 2014, in Protostars and Planets VI, ed. H. Beuther et al. (Tucson, AZ: Univ. Arizona Press), 643

Hinz, P., Bailey, V. P., Defrère, D., et al. 2014, Proc. SPIE, 9146, 91460T

Kenyon, S. J., Gómez, M., \& Whitney, B. A. 2008, in Handbook of Star Forming Regions, Volume I: The Northern sky, ed. B. Reipurth (ASP Monograph Publications, Vol. 4; San Francisco, CA: ASP), 405

Kenyon, S. J., \& Hartmann, L. 1995, ApJS, 101, 117

Kwon, W., Looney, L. W., \& Mundy, L. G. 2011, ApJ, 741, 3

Leisenring, J. M., Skrutskie, M. F., Hinz, P. M., et al. 2012, Proc. SPIE, 8446, 84464F

Maire, A.-L., Skemer, A. J., Hinz, P. M., et al. 2015, A\&A, 576, A133

Malik, M., Meru, F., Mayer, L., \& Meyer, M. 2015, ApJ, 802, 56

Marleau, G.-D., \& Cumming, A. 2014, MNRAS, 437, 1378

Marley, M. S., Fortney, J. J., Hubickyj, O., Bodenheimer, P., \& Lissauer, J. J. 2007, ApJ, 655, 541

Marois, C., Lafrenière, D., Doyon, R., Macintosh, B., \& Nadeau, D. 2006, ApJ, 641,556

Men'shchikov, A. B., Henning, T., \& Fischer, O. 1999, ApJ, 519, 257

Racine, R., Walker, G. A. H., Nadeau, D., Doyon, R., \& Marois, C. 1999, PASP, 111, 587

Rieke, G. H., \& Lebofsky, M. J. 1985, ApJ, 288, 618

Sargent, A. I., \& Beckwith, S. V. W. 1991, ApJL, 382, L31

Skemer, A. J., Hinz, P., Esposito, S., et al. 2014, Proc. SPIE, 9148, 91480L

Skrutskie, M. F., Jones, T., Hinz, P., et al. 2010, Proc. SPIE, 7735, 77353H

Soummer, R., Pueyo, L., \& Larkin, J. 2012, ApJL, 755, L28

Zhu, Z., Stone, J. M., Rafikov, R. R., \& Bai, X.-n 2014, ApJ, 785, 122 